Original Research

\title{
Which Hop Tests Can Best Identify Functional Limb Asymmetry in Patients 9-12 Months After Anterior Cruciate Ligament Reconstruction Employing a Hamstrings Tendon Autograft?
}

\author{
Jay R Ebert, PhD ${ }^{1}$, Luke Du Preez, MSc ${ }^{2}$, Bonnie Furzer, PhD², Peter Edwards, MSc ${ }^{3}$, Brendan Joss, PhD $^{4}$ \\ 1 School of Human Sciences (Exercise and Sport Science), University of Western Australia; HFRC, ${ }^{2}$ School of Human Sciences (Exercise and Sport \\ Science), University of Western Australia, ${ }^{3}$ HFRC; School of Allied Health, Curtin University, ${ }^{4}$ HFRC \\ Keywords: anterior cruciate ligament reconstruction, limb symmetry index, single limb hop test
}

https://doi.org/10.26603/001c.21140

\section{International Journal of Sports Physical Therapy}

Vol. 16, Issue 2, 2021

\section{Background}

Hop tests are commonly employed to evaluate functional limb symmetry after anterior cruciate ligament reconstruction (ACLR).

\section{Purpose}

To investigate the ability of eight hop tests to identify functional limb asymmetry in patients after ACLR.

\section{Study Design}

Prospective cohort.

\section{Methods}

Fifty patients were assessed 9-12 months following ACLR. Functional performance on both the operated and non-operated limb was assessed via eight hop tests, assessed in a randomised order. These included the: single (SHD), triple (THD) and triple crossover (TCHD) hop for distance, 6m timed hop (6MTH), single medial (MHD) and single lateral (LHD) hop for distance, single countermovement jump (SLCMJ) and timed speedy hop (TSHT). Differences in Limb Symmetry Indices (LSIs) across hop tests were compared, while Pearson's correlations were undertaken to investigate the significance and strength of the association between hop test LSIs.

\section{Results}

Significant differences were observed across hop LSIs ( $<<0.0001)$. Mean LSIs for the SHD (95.0\%), 6MTH (95.0\%), THD (96.1\%) and TCHD (95.3\%) were $\geqslant 90 \%$ and significantly greater $(\mathrm{p}<0.05)$ than the MHD (87.3\%), LHD (87.5\%), SLCMJ (83.4\%) and TSHT (86.5\%), which were all $<90 \%$. The LSI for the SLCMJ was significantly lower $(p<0.05)$ than all other hop tests. While significant correlations existed across the majority of hop LSIs, the strongest correlations existed between the SHD, THD and TCHD ( $\mathrm{r}=0.70-0.80)$, and lowest correlations between the TSHT and the other hop tests $(r=0.26-0.49)$.

\section{Conclusions}

The LHD, MHD and TSHT, as well as the SLCMJ in particular, were best able to

\footnotetext{
a Corresponding Author:

Dr Jay R. Ebert

The School Human Sciences (M408)

The University of Western Australia

35 Stirling Highway

Crawley, 6009, Western Australia

Phone: +61-8-6488-2361

Fax: +61-8-6488-1039

E-mail: jay.ebert@uwa.edu.au
} 
demonstrate functional limb asymmetry in patients following ACLR. These hop measures should be incorporated into hop test batteries, if the purpose is to detect the presence of lingering functional deficits.

\section{Level of Evidence}

Level 3.

\section{INTRODUCTION}

While approximately $90 \%$ of patients that experience an anterior cruciate ligament (ACL) tear undergo ACL reconstruction (ACLR), ${ }^{1}$ Ardern et al. reported that only $65 \%$ of patients return to their pre-injury level of sport. ${ }^{2}$ Overall, following ACLR it has been reported that approximately $7 \%$ may sustain an ipsilateral re-tear and a further $8 \%$ a contralateral ACL tear, ${ }^{3,4}$ with young athletes $(<25$ years) that return to sport (RTS) demonstrating a combined secondary ACL injury rate of $23 \% .{ }^{4}$ While a range of factors may contribute to ACL re-tears, 1,5,6 the patient's inability to regain muscle function and strength, both critical elements required in the safe RTS, have also been reported as key risk factors for re-tear. ${ }^{7,8}$ Consequently, physical performance testing is widely used in clinical settings to assess lower limb strength and functional symmetry, and research has demonstrated an increased re-injury risk if patients RTS without meeting certain strength and hop test performance cut-offs. ${ }^{9,10}$

Functional hop tests have been commonly employed in the assessment of patients after ACLR, ${ }^{9-11}$ often reported via a Limb Symmetry Index (LSI). The LSI is a measure of the operated limb as a percentage of the non-operated limb, and literature has suggested that an LSI $\geqslant 90 \%$ is considered to be 'normal' when comparing the ACLR limb to the nonoperated limb. ${ }^{12,13}$ While LSIs are commonly employed to present functional outcomes, ${ }^{13-15}$ it is unknown how applicable this reported $90 \%$ cut-off is to different hop tests, while it has been reported that LSIs can overestimate knee function in patients following ACLR, particularly given deconditioning that can occur in the comparative, non-operated limb. ${ }^{16}$ Despite their widespread use, many of these single limb hop tests are straight line movements and have been criticized for not sufficiently evaluating functional performance in patients following ACLR, ${ }^{17}$ with a recent study suggesting some hop tests may be more sensitive in detecting side-to-side differences in functional performance. ${ }^{18}$ Nonetheless, these distance- and time-base performance hop tests remain a convenient and inexpensive means of assessing higher-level functional ability, are practical in a clinical setting without excessive space nor equipment required, and provide a quantifiable measure that can be assessed over time. Of course, the decision to progress rehabilitation, increase training loads and return to agility and/or sport-specific training (and eventually sport) is dependent on more than hop testing (and a hop test battery), including measures of isokinetic muscle strength assessment, physical fitness and biomechanical movement, as well as psychological readiness. However, an investigation into the array of hop tests is warranted to determine which may be a better reflection of function (and functional deficit) in patients following ACLR. While a battery of hop measures (rather than a single hop test) is recommended in the evaluation of patients after ACLR, ${ }^{19}$ the therapist must be confident that the hop tests they include in such a battery are able to assess higher level functional capacity, especially if their combined purpose is to detect the presence of lingering functional deficits.

Therefore, the primary purpose of the current study was to investigate the ability of eight hop tests to identify functional limb asymmetry in patients after ACLR. Firstly, it was hypothesized that significant variation in lower limb symmetry measures would exist across the eight functional hop tests employed, and those incorporating multi-directional movement would demonstrate greater asymmetry. Secondly, it was hypothesized that the strongest correlations between hop tests would exist for forwards distancebased hop measures, with the weakest correlations observed in those incorporating multi-directional movement.

\section{METHODS}

\section{PARTICIPANTS}

This cross-sectional study was undertaken in a consecutive series of 50 patients (34 males, 16 females) who had undergone primary ACLR, undertaken by one of five consultant orthopaedic surgeons within the private hospital system (each with $\geqslant 10$ years of experience undertaking ACLR as a private consulting surgeon). Patients were assessed between January and August 2019, and were included if they were: 1) 16-50 years of age, 2) 9-12 months post-surgery, 3) had undergone ACLR utilising a hamstrings autograft harvested from the ipsilateral knee, 4) were experiencing no ongoing problems with either the operated or non-operated knee (inclusive of both pain and/or perceived function during their own activities of daily living), 5) did not report any other musculoskeletal concerns with pain, symptoms and/ or dysfunction in the ipsilateral or contralateral limb (that would affect their ability to perform in the hop measures), 6) had no prior recollection of significant injury or surgery on the non-operated (contralateral) limb, and 7) had returned (or were planning on returning) to Level 1 (participation 4-7 days/week) or Level 2 (participation 1-3 days per week) sports that included jumping, hard pivoting, cutting, running, twisting and/or turning sports, as reported by the Noyes Sports Activity Rating Score (NSARS). ${ }^{20}$ The cohort included 42 (84\%) and 8 (16\%) patients that ruptured their ACL in a non-contact and contact situation, respectively. Patients undergoing concomitant meniscectomy $(\mathrm{n}=10)$ or meniscal repair $(\mathrm{n}=14)$ were included.

\section{FUNCTIONAL HOP TEST BATTERY}

Patients underwent a standardized warm up, consisting of five minutes at low resistance on an upright stationary bike at a self-determined intensity, followed by an optional stretching period of 5-10 minutes which was not standard- 
ized and dictated by the patients preferred warm up routine. Patients then completed eight functional hop tests (two timed and six distance/height hop tests), undertaken in a pre-determined randomized order. Patients were given verbal descriptions of each hop test prior to undertaking each and were initially permitted two to three warm-up hops on each limb prior to initiating the hop battery. Each test was initiated on the non-operated limb, alternating between the non-operated and operated limbs until the designated number of valid test trials were obtained for each. While the hop battery was randomized in an attempt to mitigate any fatigue affects over time, the rest period between the hop trials was not standardized and was dictated by the patient's perceived readiness to proceed (that is, rather than setting a pre-determined rest period within each hop trial, an attempt to 'standardize' for each patient was made by ensuring that the patient could proceed when they deemed ready).

The eight functional hop tests included the: 1) Single Hop for Distance (SHD), 2) 6m Timed Hop (6MTH), 3) Triple Hop for Distance (THD), 4) Triple Crossover Hop for Distance (TCHD), 5) Single Medial Hop for Distance (MHD), 6) Single Lateral Hop for Distance (LHD), 7) Single Limb Countermovement Jump for Height (SLCMJ), and 8) Timed Speedy Hop Test (TSHT). For the SHD, patients were advised to hop off one limb as far as possible (forwards), landing on the same limb in a controlled manner (for this study, a controlled landing was defined as the patient's ability to cleanly hold or 'stick' the landing without any observed shuffle or stutter, and without touching down with the contralateral limb or any other part of the body) (Figure 1). The $6 \mathrm{MTH}$ involved multiple consecutive single limb hops on the same limb over a distance of $6 \mathrm{~m}$ (Figure 2). A stopwatch was used to determine the time taken to cover the distance, and the patient was instructed to hop over the designated distance in as little time as possible, treating the $6 \mathrm{~m}$ mark as a finish line without the need to land in a controlled manner. For the THD, patients had to perform three consecutive hops for maximum distance in a forward direction (all on the same limb, and without pausing in between each hop with the exception of the final landing), with a controlled landing on the third and final hop (Figure 3). For the TCHD, similar to the THD patients had to perform three consecutive hops for maximum distance, though crossing back and forth over a custom-made $15 \mathrm{~cm}$ width mat, without touching the mat (Figure 4). For both the SHD, THD and TCHD, the trial was considered successful if the patient landed in a controlled manner on the final hop.

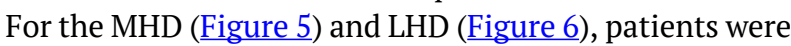
instructed to hop sideways in a medial or lateral direction, respectively, as far as possible. The hop was again considered successful if the patient landed in a controlled manner, though with the foot landing parallel to its starting position. For the SLCMJ, patients were asked to hop off one leg as high as possible (Figure 7). In order to assess jump height, the SLCMJ test employed an accelerometer (Myotester, Myotest S.A., Sion, Switzerland) which was fixed firmly around the waist using a Velcro strap, immediately superior to the greater trochanter. ${ }^{18}$ Finally, the TSHT required the patient to hop throughout a 16-hop agility course (that included forwards, backwards and sideways di-

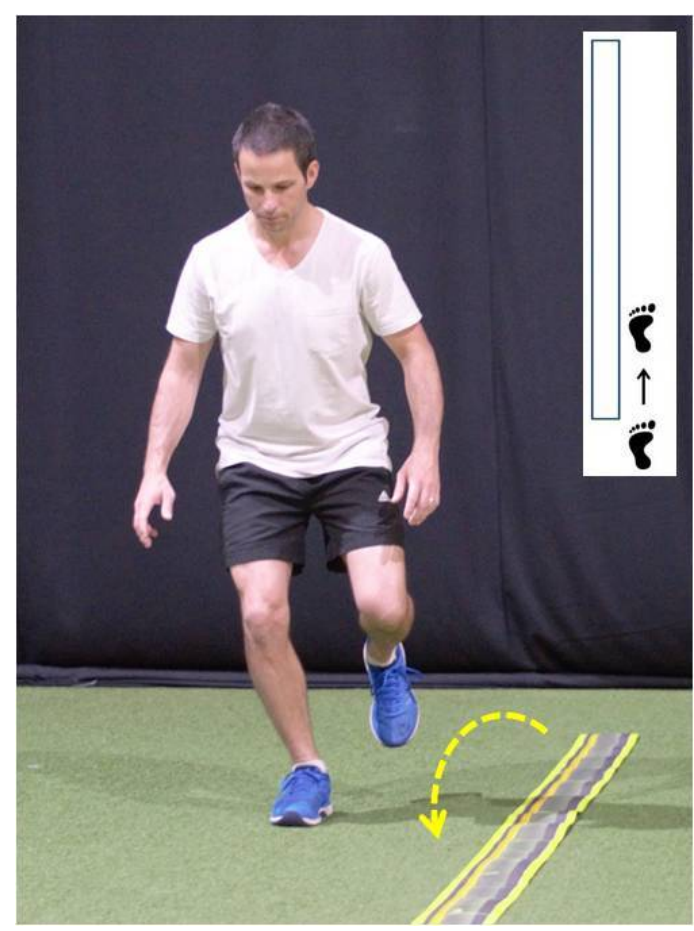

Figure 1: The Single Hop for Distance (SHD) Test, whereby the patient had to hop off one leg as far as possible, landing in a controlled manner on the same limb.

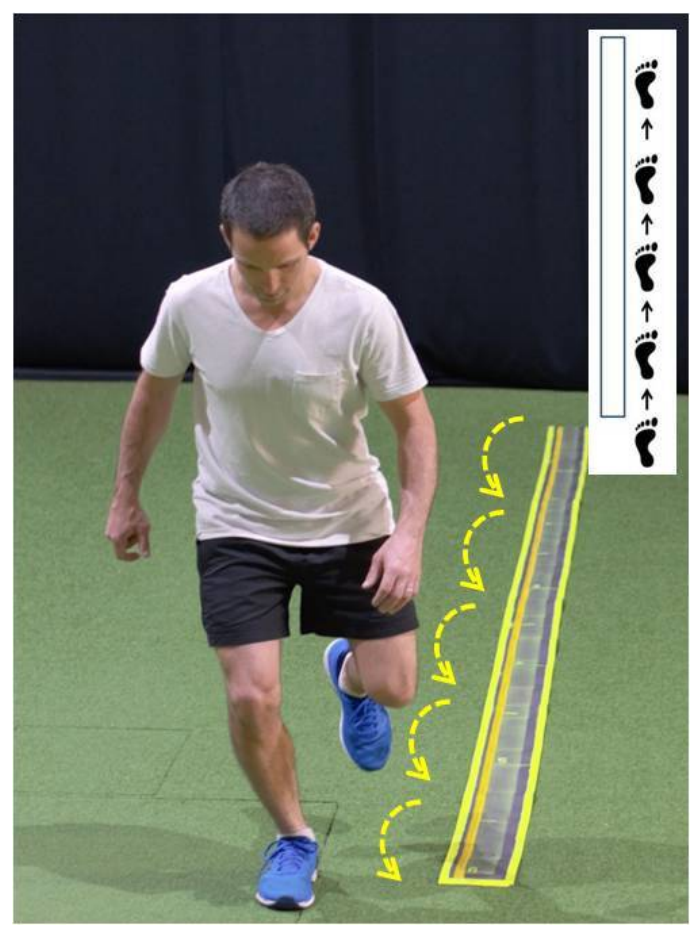

Figure 2: The 6m Timed Hop (6MTH) Test, whereby the patient had to hop on one limb as fast as possible over a distance of 6 metres.

rection hops) as fast as possible (Figure 8). A stopwatch was used to determine the time taken to cover the course, 
which was made utilising the Speedy Basic Jump Set (TST Trendsport, Grosshoflein, Austria). A total of three valid hop trials was required for the SHD, MHD, LHD, THD, TCHD and SLCMJ, while two valid hop trials were required for the $6 \mathrm{MTH}$ and TSHT. Furthermore, free use of the arms was permitted during all hop tests for consistency.

\section{DATA AND STATISTICAL ANALYSIS}

Initially, the best score (distance, height or time) for each hop test, on each limb, was employed in the final analysis. Using this best score, LSIs were obtained for each of the eight hop tests, calculated by dividing the peak values on the operated limb by that recorded on the non-operated limb for all distance/height measures (and by dividing the scores on the non-operated limb by that recorded on the operated limb for the timed measures). For the entire cohort $(n=50)$ as well as specifically for males $(n=34)$ and females $(\mathrm{n}=16)$, the LSI means (SD) and ranges were reported, together with the number (and percentage) of patients demonstrating LSIs $\geqslant 90 \%$ for each hop measure. Analysis of Variance (ANOVA) was employed to evaluate differences in LSIs across all of the functional hop measures, and post-hoc t-tests were employed to investigate where differences existed. Independent t-tests were employed to evaluate any LSI differences in hop measures between males and females. Pearson's correlations were undertaken to investigate the significance (at the $\mathrm{p}<0.01$ and $\mathrm{p}<0.05$ level) and strength of the association between LSIs for each of the eight varied hop tests. Statistical analyses were undertaken using SPSS version 23.0 (SPSS Inc, Chicago, Illinois, USA). Statistical significance was determined at $\mathrm{p}<0.05$, while the size of the Pearson's correlations were reported according to Mukaka (0.0-0.3 negligible, 0.3-0.5 low, 0.5-0.7 moderate, 0.7-0.9 high, 0.9-1.0 very high). ${ }^{21}$

An a priori sample size power calculation based on detecting statistically significant differences across the eight hop measures was determined using G-Power (Dusseldorf, Germany). Based on preliminary pilot data undertaken in 20 patients comparing LSIs across the eight functional hop tests assessed as part of the current study, a moderate effect size $(\mathrm{d}=0.56)$ was estimated. Therefore, in order to detect significant differences in mean LSIs across the eight hop tests, a minimum sample of $n=8$ was estimated to reveal differences at alpha 0.05 with $80 \%$ power. Ethics was attained from the Hollywood Private Hospital Human Research Ethics Committee (HPH382) prior to patient recruitment and evaluation. Informed consent was received and the rights of the subjects were protected.

\section{RESULTS}

The mean age of the recruited cohort was 28.3 (SD 9.1, range 16-47) years with a mean body mass index of 24.6 (SD 2.8, range 18.8-31.4). At the time of review, patients were on average 10.2 (SD 1.4, range 9-12) months post-surgery, and the mean time from injury to their primary ACLR was 10.4 (SD 11.9, range 2-54) weeks.

Table 1 shows the mean (SD) LSI and range for each of the eight hop tests undertaken, for the entire cohort as well as specifically for males and females. For the entire

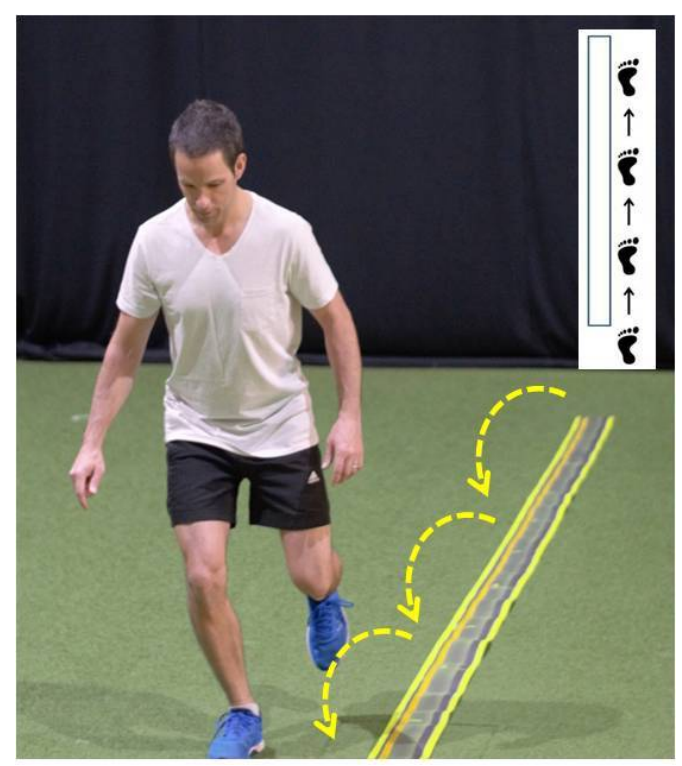

Figure 3: The Triple Hop for Distance (THD) Test, whereby the patient had to undertake three consecutive hops in a forwards direction, landing in a controlled manner on the third hop.

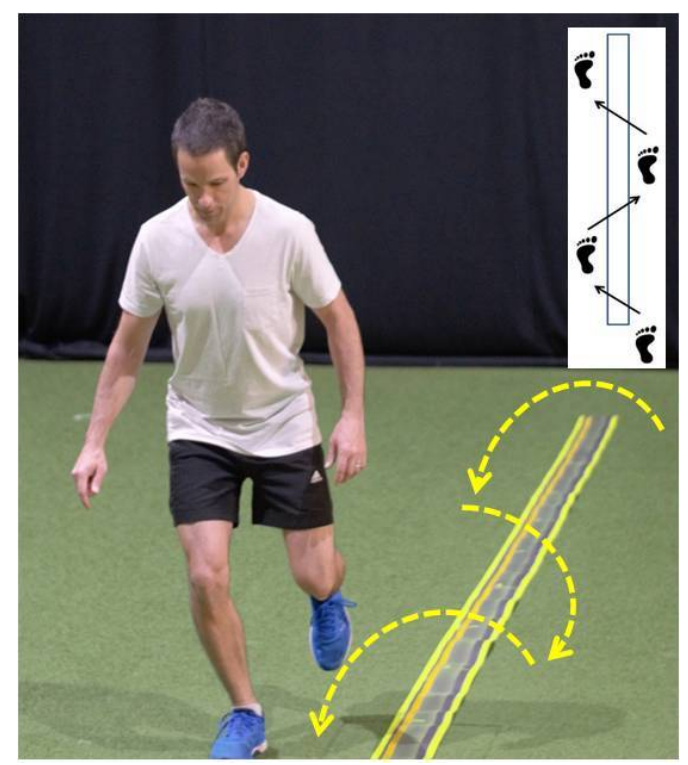

Figure 4: The Triple Crossover Hop for Distance (TCHD) Test, whereby the patient had to undertake three consecutive hops alternating back and forth over a $15 \mathrm{~cm}$ width mat (without touching the mat) in a forwards direction, landing in a controlled manner on the third hop.

cohort, ANOVA demonstrated significant differences across the eight hop tests $(\mathrm{p}<0.0001)$. The mean LSIs for the SHD (95.0\%), 6MTH (95.0\%), THD (96.1\%) and TCHD (95.3\%) were all $\geqslant 90 \%$, and not significantly different $(p>0.05)$ from each other. These were all significantly greater $(p<0.05)$ than the mean LSIs observed for the MHD (87.3\%), LHD (87.5), SLCMJ (83.4\%) and TSHT (86.5\%), which were all 
$<90 \%$ (Table 1). While the LSIs for the MHD, LHD and TSHT were not significantly different from each other $(p>0.05)$, the mean LSI for the SLCMJ was significantly lower $(\mathrm{p}<0.05)$ than every other hop test. There were no significant differences $(>0.05)$ for any of the hop LSIs between males and females.

While the majority of patients demonstrated an LSI $\geqslant 90 \%$ for the SHD (88\%), 6MTH (80\%), THD (88\%) and TCHD
(84\%), the majority were $<90 \%$ for the MHD (46\%), LHD (30\%), SLCMJ (18\%) and TSHT (32\%) (Table 1). Overall, 36 patients $(72 \%)$ demonstrated an LSI $\geqslant 90 \%$ for the combined hop battery of the SHD, 6MTH, THD and TCHD. Only five patients demonstrated an LSI $\geqslant 90 \%$ for every one of the eight hop tests. 
Table 1: Limb Symmetry Indices (LSIs) for each of the eight hop tests undertaken, across the 50 patients assessed as well as specifically for males (n=34) and females

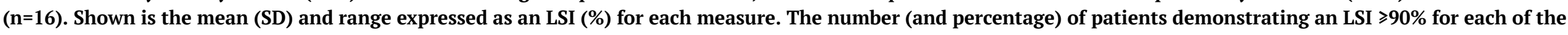
hop tests is also shown.

\begin{tabular}{|c|c|c|c|c|c|c|c|c|c|}
\hline \multirow[b]{2}{*}{ Test } & \multicolumn{3}{|c|}{ Entire Cohort $(\mathrm{n}=50)$} & \multicolumn{3}{|c|}{ Males $(n=34)$} & \multicolumn{3}{|c|}{ Females $(n=16)$} \\
\hline & Mean (SD) & Range & $\geq 90 \%$ LSI, n (\%) & Mean (SD) & Range & $\geq 90 \%$ LSI, n (\%) & Mean (SD) & Range & $\geq 90 \%$ LSI, n (\%) \\
\hline SHD & $95.0(5.0)$ & $81.9-105.2$ & $44(88)$ & $94.9(4.8)$ & $81.9-101.8$ & $30(88)$ & $95.3(5.6)$ & $84.6-105.2$ & $14(88)$ \\
\hline $6 \mathrm{MTH}$ & $95.0(7.5)$ & 75.4-116.2 & $40(80)$ & $95.0(7.4)$ & $76.4-116.2$ & $27(79)$ & $95.1(7.8)$ & $75.4-104.6$ & $13(81)$ \\
\hline THD & $96.1(6.3)$ & 77.1-106.5 & $44(88)$ & $96.2(6.2)$ & $77.1-106.5$ & $30(88)$ & $95.9(6.7)$ & $78.4-105.3$ & $14(88)$ \\
\hline TCHD & $95.3(6.6)$ & $79.4-104.9$ & $42(84)$ & $94.7(6.5)$ & $79.4-103.4$ & $29(85)$ & $96.5(6.8)$ & $80.6-104.9$ & $13(81)$ \\
\hline LHD & $87.5(6.4)$ & $64.0-97.7$ & $15(30)$ & $87.7(6.7)$ & $64.0-96.6$ & $11(32)$ & $87.1(6.1)$ & $73.1-97.7$ & $4(25)$ \\
\hline MHD & $87.3(7.3)$ & $62.9-100.0$ & $23(46)$ & $87.3(7.6)$ & $62.9-100.0$ & $17(50)$ & $87.3(7.1)$ & $73.2-100.0$ & $6(38)$ \\
\hline SLCMJ & $83.4(9.5)$ & $62.2-104.2$ & $9(18)$ & $83.8(8.8)$ & 63.1-99.7 & $6(18)$ & $82.5(11.2)$ & $62.2-104.2$ & $3(19)$ \\
\hline TSHT & $86.5(8.9)$ & $64.0-101.3$ & $16(32)$ & $87.6(7.8)$ & $67.8-98.1$ & $11(32)$ & $84.3(11.0)$ & $64.0-101.3$ & $5(31)$ \\
\hline
\end{tabular}

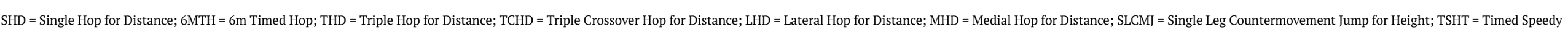
Hop Test. 
Table 2: Pearson correlations between the eight hop tests.

\begin{tabular}{|c|c|c|c|c|c|c|c|c|}
\hline & SHD & $6 \mathrm{MTH}$ & THD & TCHD & MHD & LHD & SLCMJ & TSHT \\
\hline SHD & 1.00 & & & & & & & \\
\hline $6 \mathrm{MTH}$ & $0.46^{* *}$ & 1.00 & & & & & & \\
\hline THD & $0.71^{* *}$ & $0.43^{* *}$ & 1.00 & & & & & \\
\hline $\mathrm{TCHD}$ & $0.70^{* *}$ & $0.48^{* *}$ & $0.80^{* *}$ & 1.00 & & & & \\
\hline MHD & $0.50^{* *}$ & $0.44^{* *}$ & $0.46^{* *}$ & $0.38^{* *}$ & 1.00 & & & \\
\hline LHD & $0.59^{* *}$ & $0.51^{* *}$ & $0.53^{* *}$ & $0.62^{* *}$ & $0.63^{* *}$ & 1.00 & & \\
\hline SLCMJ & $0.52^{* *}$ & $0.55^{* *}$ & 0.20 & $0.55^{* *}$ & $0.52^{* *}$ & $0.51^{* *}$ & 1.00 & \\
\hline TSHT & $0.29^{*}$ & $0.29^{* *}$ & $0.49^{* *}$ & 0.26 & $0.31^{*}$ & $0.39^{* *}$ & $0.43^{* *}$ & 1.00 \\
\hline
\end{tabular}

SHD = Single Hop for Distance; $6 \mathrm{MTH}$ = 6m Timed Hop; THD = Triple Hop for Distance; TCHD = Triple Crossover Hop for Distance; MHD = Medial Hop for Distance; LHD = Lateral Hop for Distance; SLCMI = Single Leg Countermovement Jump for Height; TSHT = Timed Speedy Hop Test.

* Correlation is significant at the 0.05 level (2-tailed)

*** Correlation is significant at the 0.01 level (2-tailed)

Statistically significant $(\mathrm{p}<0.05)$ and positive correlations existed across the majority of hop tests (Table 2). High positive correlations ( $r=0.70-0.80)$ existed between the SHD, THD and TCHD. Low and moderate positive correlations were observed between the TSHT $(r=0.26-0.49)$, SLCMJ $(\mathrm{r}=0.20-0.55)$, MHD ( $\mathrm{r}=0.38-0.63)$ and LHD ( $\mathrm{r}=0.39-0.63)$, with each of the other hop tests (Table 2).

\section{DISCUSSION}

The most important finding of the current study was that some hop measures (LHD, MHD, SLCMJ and TSHT) demonstrated significantly lower mean LSIs than others (SHD, $6 \mathrm{MTH}, \mathrm{TCD}$ and TCHD). The majority of patients achieved $\geqslant 90 \%$ LSI scores on the four more commonly reported hop tests (SHD, 6MTH, THD, TCHD). In contrast, the majority of patients demonstrated LSIs $<90 \%$ on the four lesser reported hops (LHD, MHD, SLCMJ, TSHT).

Recent studies have demonstrated an increased re-injury risk if patients RTS without meeting $\geqslant 90 \%$ LSI cut-offs, in a range of hop and strength parameters undertaken as part of a test battery. ${ }^{9,10}$ A four times greater re-injury risk in those who RTS after ACLR was reported by Kyritsis et al. if not meeting the RTS discharge criteria that included an LSI $>90 \%$ in the SHD, THD and TCHD. ${ }^{10}$ Grindem et al. also reported a greater re-injury rate if LSIs $>90 \%$ for quadriceps strength and functional hop symmetry were not met, which included the aforementioned three hop measures, as well as the 6MTH. ${ }^{9}$ These four hop measures have been reported previously in the form of a hop test battery, ${ }^{11}$ and the SHD has been reported as one of the most common functional hop measures reported for use in patients after ACLR. ${ }^{22}$ A systematic review undertaken by Hegedus et al. reported that despite numerous published studies employing hop tests such as the SHD, 6MTH, THD and TCHD, there remains limited and conflicting evidence regarding the reliability, agreement, construct validity, criterion validity and responsiveness of these functional measures. ${ }^{23}$

Furthermore, it should be acknowledged that despite their widespread use, many of these single limb hop tests are straight line movements (apart from the TCHD which

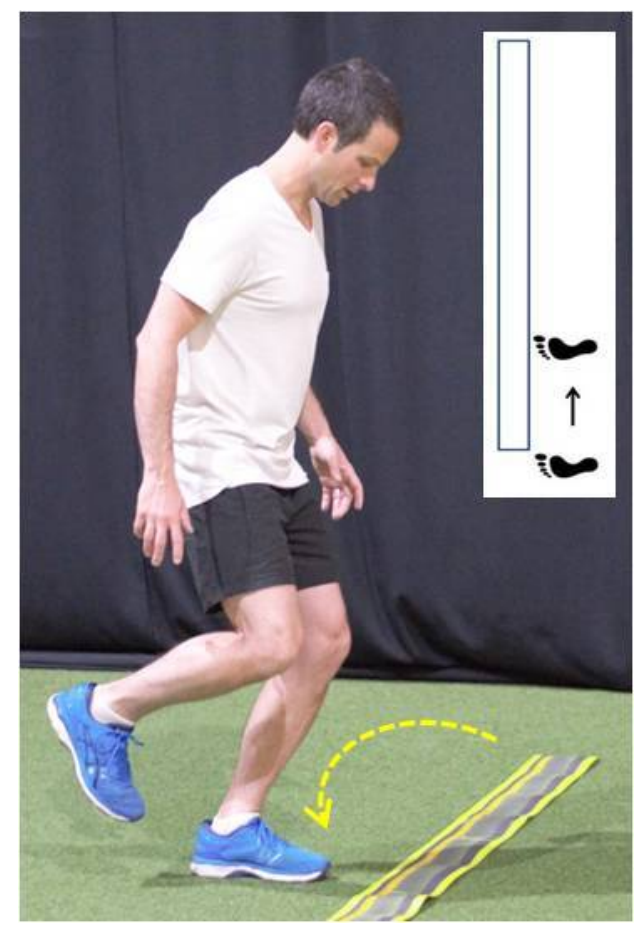

Figure 5: The Single Medial Hop for Distance (MHD) Test, whereby the patient had to hop off one leg in a medial direction as far as possible, landing in a controlled manner on the same limb.

incorporates some multi-directional, albeit not hard cutting hops) and have been criticized for not sufficiently evaluating functional performance in patients following ACLR. ${ }^{17}$ A recent study by Ebert et al. reported that the TSHT and SLCMJ demonstrated significantly lower mean LSIs in patients after ACLR than the more commonly employed and reported SHD, 6MTH, THD and TCHD, suggesting some hop tests may be more sensitive in detecting side-to-side differences in functional symmetry. ${ }^{18}$ The TSHT requires multiple forwards, sideways and backwards hops combined as a timed test, which may prove more challenging for patients (hence the lower mean LSIs observed). Admittedly, the ear- 
lier Ebert et al. ${ }^{18}$ study did not permit free use of the arms for the SLCMJ as was the case in the current study, though having to propel the body upward against gravity, as opposed to the forwards direction, may still require more demand (strength and power) from the quadriceps muscles to generate height. Finally, the reasons for the significantly lower LSIs on the MHD and LHD can only be speculated. While both require a controlled land and move in side directions which is different to the more traditionally employed hop tests, ${ }^{11}$ anecdotally, it appears harder to generate the power required during the LHD to transition laterally (i.e. factors such as arm swing are better controlled for, unlike the forwards hop measures). The MHD may require a more challenging landing position, whereby the momentum moving medially almost forces the lower limb into a more dynamic valgus position, which may then be harder to land in patients lacking adequate lower limb muscular control. Finally, it should be acknowledged that both of these side hop measures may simply present relatively naïve tasks for the patient. This may create a higher degree of difficulty, thereby presenting greater asymmetry and highlighting potential benefit in their utility. The first hypothesis was that significant variation in lower limb symmetry measures would exist across the eight functional hop tests employed, and those incorporating multi-directional movement would demonstrate greater asymmetry. Overall, the first hypothesis was largely supported.

The current study also investigated the correlation between LSIs across each of the functional hop tests. While statistically significant and positive correlations existed across the majority of hop tests, the strength of these associations varied. The strongest correlations existed between the SHD, THD and TCHD, all distance-based measures incorporating forwards and straight-line movements. The second hypothesis was that the strongest correlations between hop tests would exist for forwards distance-based hop measures, with the weakest correlations observed in those incorporating multi-directional movement. Therefore, this partially supported the second hypothesis and, combined with the similar mean LSIs observed for each of these hop tests, would suggest that the use of any of these three hop tests would provide similar information to the clinician if the presence of functional limb asymmetry was the primary concern. The TSHT, SLCMJ, MHD and LHD demonstrated the weakest correlations with the other hop tests. As outlined above, the TSHT is a timed measure incorporating multi-directional movements which would also support the second hypothesis. While the MHD and LHD are not multi-directional measures, they are also not hop measures in a forwards direction, and it unknown whether this provides rationale for the weaker correlation with the forwards distance tests given they are directions less encountered during activities of daily living.

It is important to note that the current study employed a range of hop measures commonly reported in the literature and/or used routinely through our clinical institution. However, a range of other hop tests have been reported in addition to those employed in the current study including, though not limited to, the triple medial hop for distance, ${ }^{24}$ the $90^{\circ}$ medial rotation hop for distance, ${ }^{24}$ the single timed lateral hop ${ }^{25}$ and 30 s side hop test, ${ }^{15}$ the timed

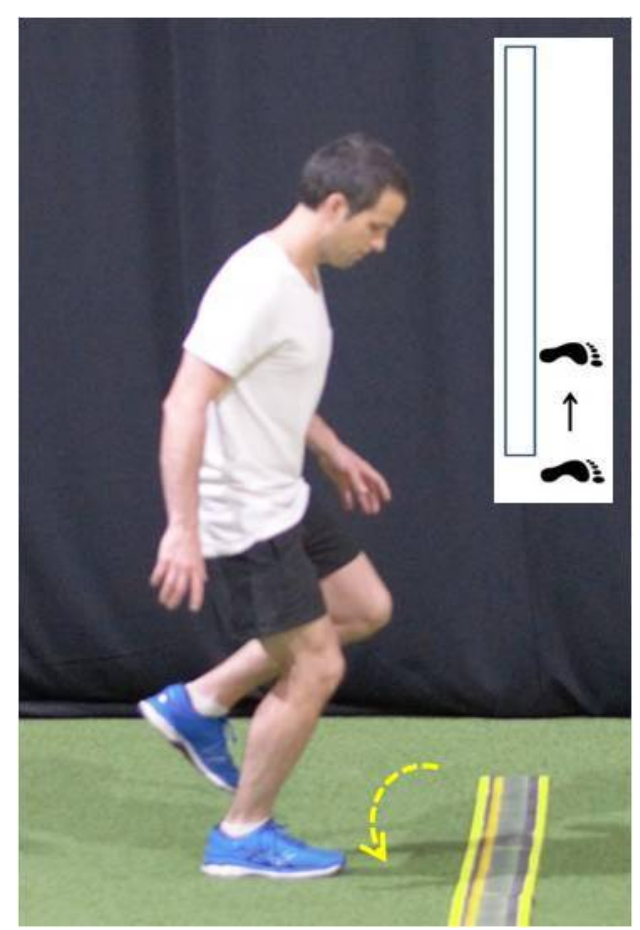

Figure 6: The Single Lateral hop for Distance (LHD) Test, whereby the patient had to hop off one leg in a lateral direction as far as possible, landing in a controlled manner on the same limb.

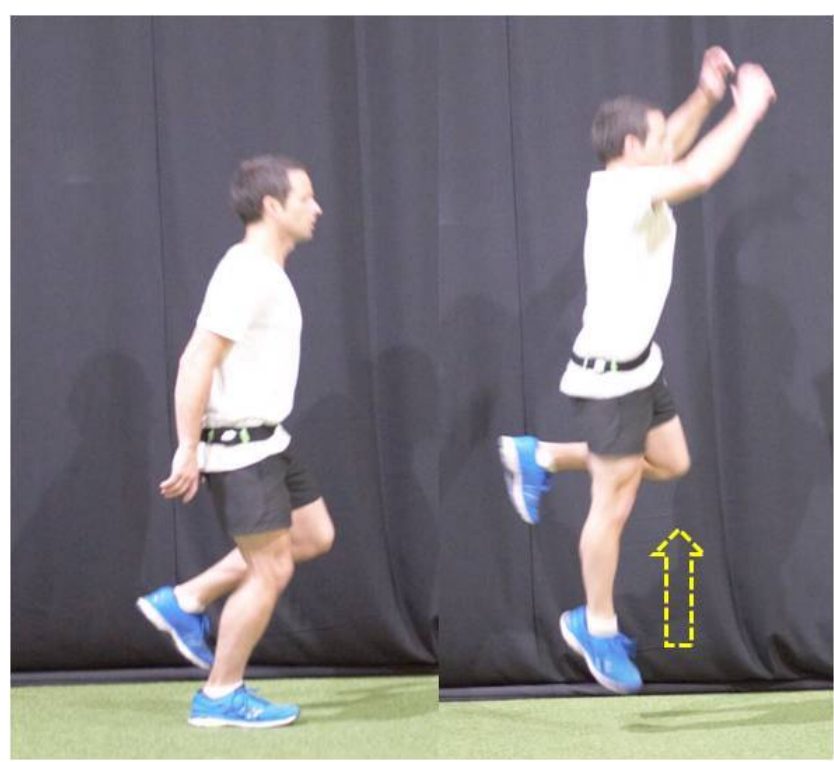

Figure 7: The Single Leg Countermovement Jump (SLCMJ) for Height, whereby the patient had to hop off one leg as high as possible, landing in a controlled manner on the same limb.

square hop, ${ }^{15}$ the figure-of-eight timed hop test, ${ }^{26}$ and a drop jump followed by a double hop for distance. ${ }^{15}$ While we sought to manage the role of fatigue via hop test randomisation and providing patients the rest time they felt they required, incorporating more hop tests may have jeop- 
ardized this. These other reported hop tests warrant further investigation, given the results of the current study.

Furthermore, it should also be acknowledged that $\geqslant 90 \%$ LSI cut-offs are often employed in patients following $\operatorname{ACLR}^{9,10}$ for more traditional hop tests (including the SHD, 6MTH, THD and TCHD), largely when considering that normative data in healthy subjects (comparing the dominant and non-dominant limb) demonstrates mean LSIs in these hop tests ranging from $98-102 \% .^{27}$ However, while this study again sought to compare LSIs across the varied hop tests, we are yet to appreciate what LSI may be considered 'normal' or 'abnormal' for all of these hop measures that lack normative data in a healthy population. While healthy normative mean LSIs of 101-104\% (dependent on gender and age) have been reported for the $\mathrm{TSHT}^{3}$, mean LSIs for the SLCMJ have been reported to range from 102-124\% in favour of the dominant limb (albeit this was undertaken with fixed arms, rather than free use of arms as permitted in the current study). ${ }^{3}$ To the best of the author's knowledge, normative LSIs in a healthy population having been presented for the MHD or LHD. Developing a normative dataset for all hop measures remains an area for future research, in order to better appreciate the LSIs observed in these other hop tests in the post-operative ACLR cohort.

The current study acknowledges a number of further limitations. Firstly, the lack of study generalizability should be acknowledged given the pre-defined cohort that was employed, including those undergoing ACLR via a hamstring autograft, those within 16-50 years of age and those specifically 9-12 months post-surgery. The surgical graft method and age range was set to accommodate the majority of patients seen in clinical practice locally, as well as those most likely to be seeking a RTS (which also served as part of the study inclusion criteria). We sought to evaluate patients at 9-12 months given anecdotally this has traditionally been a time that patients transition down a RTS pathway, while existing evidence has reported a reduced re-injury rate in patients returning to pivoting sports after 9 months postsurgery. ${ }^{9}$ Secondly, while LSIs are commonly employed to present functional outcomes, ${ }^{13-15}$ Wellstandt et al. reported these can overestimate knee function in patients following ACLR. ${ }^{16}$ Furthermore, mean LSIs can be misleading given the potential for high LSIs to balance out poor LSIs, hence the current study also presented the amount of patients above and below the reported 90\% LSI cut-off for each test. Nonetheless, this study sought to compare LSIs across the varied tests. Thirdly, while existing literature has suggested that an LSI $\geqslant 90 \%$ is considered to be 'normal' when comparing the ACLR limb to the non-operated limb, ${ }^{12,13}$ we are yet to ascertain how applicable this reported $90 \%$ cut-off is to different hop tests (i.e. single versus multihop measures, or straight line versus multi-directional hop tests). This requires further investigation, though the current study also sought to investigate the presence of significant differences between mean LSIs.

Fourthly, it should be acknowledged that the patients recruited for this study received varied rehabilitation guidance and exercise prescription, which could affect each patient's ability to undertake each of the varied hop measures. Furthermore, for the current study there were no specific objective criteria employed to essentially 'clear' the patient

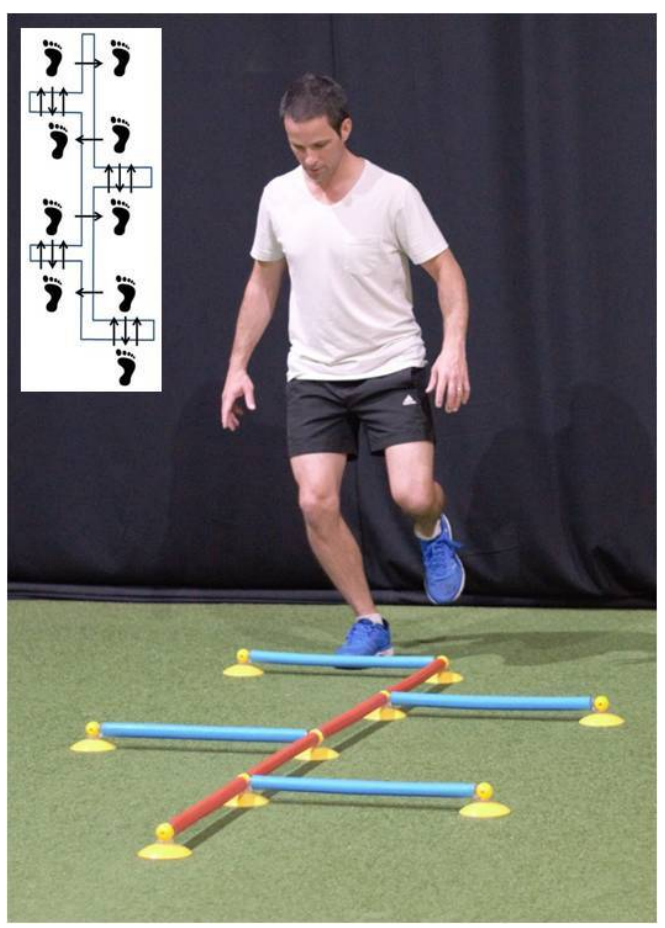

Figure 8: The Timed Speedy Hop Test (TSHT), whereby the patient had to hop on one limb as fast as possible throughout the 16-hop course that included forwards, backwards and sideways hops.

for study participation, apart from that already mentioned including time from surgery and the absence of knee and/ or musculoskeletal pain in general that may affect performance. Again, the current study sought to investigate differences across functional hop tests (within patients), rather than between patients. Fifthly, while the hop battery was randomized in an attempt to mitigate any fatigue affects, the rest period between the hop trials was not standardized and was dictated by the patient's perceived readiness to proceed. This may affect the results across patients, albeit it was decided upon to ensure that all fitness levels could be accommodated and each patient was not forced to progress before they felt ready (as would have been the case with a pre-determined rest period). Finally, it should be acknowledged that functional hop symmetry is only one part of the larger and more comprehensive RTS decision making process. Furthermore, these performance-based hop measures seek to evaluate distance or time, and lack an objective assessment of lower limb biomechanics which may be associated with the risk of secondary ipsilateral and contralateral ACL rupture. ${ }^{28}$ Welling et al. recently reported clinically relevant altered movement patterns in patients after ACLR in the SHD, despite LSIs $>90 \%,{ }^{29}$ while a recent systematic review and meta-analysis outlined the risk of using distance only during the SHD given the additional presence of several kinetic and kinematic deficits commonly observed in patients following ACLR. ${ }^{30}$

\section{CONCLUSION}

In the current study, more commonly reported and em- 
ployed functional hop measures (SHD, 6MTH, THD and TCHD) demonstrated mean LSIs $\geqslant 90 \%$, with the majority of patients $(80-88 \%)$ demonstrating an LSI $\geqslant 90 \%$ for each hop test as assessed at 9-12 months following ACLR undertaken via a hamstring tendon autograft. However, significantly lower mean LSIs (<90\%) were observed in lesser reported functional hop measures (MHD, LHD, SLCMJ and TSHT), with the majority of patients (54-78\%) demonstrating an LSI $<90 \%$ for each hop test. The current results would suggest that the latter four hop tests are better at identifying side-to-side functional asymmetry in patients following ACLR. These functional hop measures should be considered in both future research settings and the clinical environment. While a battery of hop measures (rather than a single hop test) is recommended in the evaluation of patients after ACLR, ${ }^{19}$ the therapist must be confident that the hop tests they include are indeed able to assess higher level functional capacity if purpose is to detect the presence of lingering functional deficits in these patients prior to RTS.

\section{CONFLICT OF INTEREST STATEMENT}

No benefits in any form have been received or will be received from a commercial party related to the subject of this article.

\section{SOURCES OF FUNDING}

Nil.

\section{ACKNOWLEDGEMENTS}

Nil.

\section{ETHICS}

Ethics approval was obtained by the Hollywood Private Hospital (HPH382).

Submitted: March 29, 2020 CDT, Accepted: October 10, 2020 CDT 


\section{REFERENCES}

1. Paterno MV, Rauh MJ, Schmitt LC, Ford KR, Hewett TE. Incidence of second ACL injuries 2 years after primary ACL reconstruction and return to sport. Am J Sports Med. 2014;42(7):1567-1573. doi:10.1177/03635 $\underline{46514530088}$

2. Ardern CL, Taylor NF, Feller JA, Webster KE. Fiftyfive per cent return to competitive sport following anterior cruciate ligament reconstruction surgery: an updated systematic review and meta-analysis including aspects of physical functioning and contextual factors. Br J Sports Med.

2014;48(21):1543-1552. doi:10.1136/bjsports-2013-09 $\underline{3398}$

3. Hildebrandt C, Muller L, Zisch B, Huber R, Fink C, Raschner C. Functional assessments for decisionmaking regarding return to sports following ACL reconstruction. Part I: development of a new test battery. Knee Surg Sports Traumatol Arthrosc. 2015;23(5):1273-1281. doi:10.1007/s00167-015-352 9-4

4. Wiggins AJ, Grandhi RK, Schneider DK, Stanfield D, Webster KE, Myer GD. Risk of secondary injury in younger athletes after anterior cruciate ligament reconstruction: A systematic review and metaanalysis. Am J Sports Med. 2016;44(7):1861-1876. do i:10.1177/0363546515621554

5. Lind M, Menhert F, Pedersen AB. Incidence and outcome after revision anterior cruciate ligament reconstruction: results from the Danish registry for knee ligament reconstructions. Am J Sports Med. 2012;40(7):1551-1557. doi:10.1177/036354651244600 $\underline{0}$

6. Samitier G, Marcano AI, Alentorn-Geli E, Cugat R, Farmer KW, Moser MW. Failure of anterior cruciate ligament reconstruction. Arch Bone Jt Surg. 2015;3(4):220-240.

7. Ardern CL, Webster KE, Taylor NF, Feller JA. Return to the preinjury level of competitive sport after anterior cruciate ligament reconstruction surgery: two-thirds of patients have not returned by 12 months after surgery. Am J Sports Med. 2011;39(3):538-543. doi:10.1177/0363546510384798

8. Hewett TE, Di Stasi SL, Myer GD. Current concepts for injury prevention in athletes after anterior cruciate ligament reconstruction. Am J Sports Med. 2013;41(1):216-224. doi:10.1177/0363546512459638
9. Grindem H, Snyder-Mackler L, Moksnes H, Engebretsen L, Risberg MA. Simple decision rules can reduce reinjury risk by $84 \%$ after ACL reconstruction: the Delaware-Oslo ACL cohort study. Br J Sports Med. 2016;50(13):804-808. doi:10.1136/bjsports-2016-0960 $\underline{31}$

10. Kyritsis P, Bahr R, Landreau P, Miladi R, Witvrouw E. Likelihood of ACL graft rupture: not meeting six clinical discharge criteria before return to sport is associated with a four times greater risk of rupture. $\mathrm{Br}$ J Sports Med. 2016;50(15):946-951. doi:10.1136/bjspor ts-2015-095908

11. Reid A, Birmingham TB, Stratford PW, Alcock GK, Giffin JR. Hop testing provides a reliable and valid outcome measure during rehabilitation after anterior cruciate ligament reconstruction. Phys Ther. 2007;87(3):337-349. doi:10.2522/ptj.20060143

12. Risberg MA, Holm I, Ekeland A. Reliability of functional knee tests in normal athletes. Scand J Med Sci Sports. 1995;5(1):24-28.

13. Thomee R, Kaplan Y, Kvist J, et al. Muscle strength and hop performance criteria prior to return to sports after ACL reconstruction. Knee Surg Sports Traumatol Arthrosc. 2011;19(11):1798-1805. doi:10.1007/s0016 7-011-1669-8

14. Thomee R, Neeter C, Gustavsson A, et al. Variability in leg muscle power and hop performance after anterior cruciate ligament reconstruction. Knee Surg Sports Traumatol Arthrosc. 2012;20(6):1143-1151. doi:10.1007/s00167-012-191 $\underline{2-\mathrm{y}}$

15. Gustavsson A, Neeter C, Thomee P, et al. A test battery for evaluating hop performance in patients with an ACL injury and patients who have undergone ACL reconstruction. Knee Surg Sports Traumatol Arthrosc. 2006;14(8):778-788. doi:10.1007/s00167-00 6-0045-6

16. Wellsandt E, Failla MJ, Snyder-Mackler L. Limb symmetry indexes can overestimate knee function after anterior cruciate ligament injury. J Orthop Sports Phys Ther. 2017;47(5):334-338. doi:10.2519/jospt.201 $\underline{7.7285}$

17. Narducci E, Waltz A, Gorski K, Leppla L, Donaldson $\mathrm{M}$. The clinical utility of functional performance tests within one-year post-acl reconstruction: a systematic review. Int J Sports Phys Ther. 2011;6(4):333-342. 
18. Ebert JR, Edwards P, Currie J, et al. Comparison of the 'Back in Action' test battery to standard hop tests and isokinetic knee dynamometry in patients following anterior cruciate ligament reconstruction. Int J Sports Phys Ther. 2018;13(3):389-400.

19. Kaplan Y, Witvrouw E. When is it safe to return to sport after ACL reconstruction? Reviewing the criteria. Sports Health. 2019;11(4):301-305. doi:10.117 $\underline{7 / 1941738119846502}$

20. Noyes FR, Barber SD, Mooar LA. A rationale for assessing sports activity levels and limitations in knee disorders. Clin Orthop Relat Res. 1989;(246):238-249.

21. Mukaka MM. A guide to appropriate use of correlation coefficient in medical research. Malawi Med J. 2012;24(3):69-71.

22. Barber-Westin SD, Noyes FR. Factors used to determine return to unrestricted sports activities after anterior cruciate ligament reconstruction. Arthroscopy. 2011;27(12):1697-1705. doi:10.1016/j.art hro.2011.09.009

23. Hegedus EJ, McDonough S, Bleakley C, Cook CE, Baxter GD. Clinician-friendly lower extremity physical performance measures in athletes: a systematic review of measurement properties and correlation with injury, part 1 . The tests for knee function including the hop tests. Br J Sports Med. 2015;49(10):642-648. doi:10.1136/bjsports-2014-0940 $\underline{94}$

24. Dingenen B, Truijen J, Bellemans J, Gokeler A. Test-retest reliability and discriminative ability of forward, medial and rotational single-leg hop tests. Knee. 2019;26(5):978-987. doi:10.1016/j.knee.2019.0 $\underline{6.010}$
25. Greenberg EM, Greenberg ET, Ganley TJ, Lawrence JT. Strength and functional performance recovery after anterior cruciate ligament reconstruction in preadolescent athletes. Sports Health. 2014;6(4):309-312. doi:10.1177/19417381145 $\underline{37594}$

26. Itoh H, Kurosaka M, Yoshiya S, Ichihashi N, Mizuno K. Evaluation of functional deficits determined by four different hop tests in patients with anterior cruciate ligament deficiency. Knee Surg Sports Traumatol Arthrosc. 1998;6(4):241-245. doi:1 $\underline{0.1007 / \mathrm{s} 001670050106}$

27. Munro AG, Herrington LC. Between-session reliability of four hop tests and the agility T-test. $J$ Strength Cond Res. 2011;25(5):1470-1477. doi:10.151 9/ISC.0b013e3181d83335

28. Sward P, Kostogiannis I, Roos H. Risk factors for a contralateral anterior cruciate ligament injury. Knee Surg Sports Traumatol Arthrosc. 2010;18(3):277-291. $\underline{\mathrm{d}}$ oi:10.1007/s00167-009-1026-3

29. Welling W, Benjaminse A, Seil R, Lemmink K, Gokeler A. Altered movement during single leg hop test after ACL reconstruction: implications to incorporate 2-D video movement analysis for hop tests. Knee Surg Sports Traumatol Arthrosc. 2018;26(10):3012-3019. doi:10.1007/s00167-018-489 $\underline{3-7}$

30. Kotsifaki A, Korakakis V, Whiteley R, Van Rossom $\mathrm{S}$, Jonkers I. Measuring only hop distance during single leg hop testing is insufficient to detect deficits in knee function after ACL reconstruction: a systematic review and meta-analysis. $\mathrm{Br} J$ Sports $\mathrm{Med}$. 2020;54(3):139-153. doi:10.1136/bjsports-2018-09991 $\underline{8}$ 